\title{
Análisis del estrés académico en la adolescencia: Efectos del nivel educativo y del sexo en Educación Secundaria Obligatoria
}

\section{Analyzing academic stress in adolescence: Gender and grade level effects in Compulsory Secondary Education}

Fecha de recepción: 12/11/2014

Fecha de aceptación: 2/12/2015
Rafael García-Ros

Universitat de València. Dpto. Psicología Evolutiva y de la Educación

Francisco Pérez-González

Universitat de València. Dpto. Psicología Evolutiva y de la Educación

María C. Fuentes Durán

Universitat de València. Dpto. Metodología de las Ciencias del Comportamiento

\section{resumen/abstract:}

El estudio analiza los efectos del nivel educativo y del sexo sobre el estrés académico en una muestra de 299 estudiantes de Enseñanza Secundaria Obligatoria. Los niveles superiores de estrés en todos los cursos y en ambos sexos se evidencian en sobrecarga de trabajo y realización de exámenes, así como en las preocupaciones por el futuro académico. Se observan efectos principales significativos tanto de nivel educativo como de sexo en el estrés provocado por la realización de actividades y el estudio, siendo las mujeres y el año de incorporación a la etapa donde se evidencian valores superiores. La interacción sexo*nivel educativo es significativa en sobrecarga académica y realización de exámenes, futuro académico y relaciones interpersonales, siendo las mujeres quienes presentan mayor estrés en el último curso de esta etapa educativa. Por otro lado, mientras las mujeres muestran niveles homogéneos de estrés a lo largo de los cursos, en los varones se observa una evolución decreciente. Se discuten los resultados desde la perspectiva de la investigación previa y de sus repercusiones para la intervención psicoeducativa.

The study analyzes the effects of gender and grade level on the academic stress of a sample of 299 adolescents enrolled in Compulsory Secondary Education (grades 7-10). Higher levels of stress in all grades and both sexes are shown on work overload and taking exams, as well as on concerns about their academic future. Significant main effects of both grade level and sex are observed on the stress produced by doing activities and studying, with girls and year of incorporation in secondary school showing higher values. The sex ${ }^{*}$ grade level interaction is significant on academic overload and taking exams, academic future and interpersonal relationships, with the girls presenting higher levels in 10th grade than the boys and showing homogenous levels throughout secondary school, while the boys showed a gradual decline. The results are discussed from the perspective of prior research and their importance for educational intervention.

\section{palabras clave/keywords:}

Estrés académico; Adolescencia; Educación Secundaria Obligatoria; Diferencias individuales

Academic stress; Adolescence; Secondary Education; Age differences; Sex differences 


\section{Introducción}

El estrés académico constituye un importante tópico en la investigación e intervención psicoeducativa en las últimas décadas por su elevada prevalencia en las distintas etapas del sistema educativo, por su relación con la obtención de resultados académicos deficientes y con el abandono de los estudios (Kaplan, Liu y Kaplan, 2005; Liu y Lu, 2011a), así como por su vinculación con el bienestar de los estudiantes (Byrne, Davenport y Mazanov, 2007). En los últimos años se ha incrementado el interés de la investigación sobre la temática en enseñanza secundaria y universitaria, dado que el contexto académico constituye una de las fuentes de estrés más destacada por los adolescentes (de Anda et al., 2000; Kouzma y Kennedy, 2004), y que diversos estudios destacan su notable incrementoen las sociedades occidentales(e.g., Antonson, Thorsen, Sundquist, y Sundquist, 2013).

Sin embargo, pese al amplio volumen de estudios centrados en analizar el estrés en la adolescencia en los distintos dominios y contextos vitales, todavía no resulta clara su evolución en esta etapa, así como las posibles interacciones entre contextos, patrones de desarrollo y sexo (Seiffge-Krenke, Aunola y Nurmi, 2009). Desde esta perspectiva, dados los escasos estudios que analizan la relación entre sexo, nivel educativo y estrés académico en la adolescencia (Murberg y Bru, 2004),así como su clara utilidad para el diseño de programas de prevención e intervención en este ámbito (Grant et al.,2006), este trabajo se centra en analizar los efectos del sexo y del nivel educativo sobre el estrés académico en Educación Secundaria Obligatoria -ESO-.

De este modo, aunque los estudios centrados en esta cuestión suelen coincidir en que las adolescentes manifiestan mayor estrés académico que los varones (e.g., de Anda et al., 2000; Rayle y Chung, 2007), recientes trabajos destacan la inexistencia de diferencias en función del sexo o bien señalan que las mujeres muestran valores superiores frente a algunos tipos de estresores mientras que los varones lo hacen en otros (Hankin, Mermelstein y Roesch, 2007; Liu y Lu, 2011a; Murberg y Bru, 2004; Williams y McGillicudy-De Lisi, 2000). Por otro lado, mención especial merecen los trabajos centrados en las transiciones entre etapas educativas, donde se constata la existencia de elevados niveles de estrés (Boekaerts, 2011) y que las mujeres muestran valores superiores frente a los varones (Rayle y Chung, 2007; Rice, Frederickson, y Seymour, 2011).

Los escasos estudios que analizan la evolución del estrés en función de la edad y niveles educativos tienden a señalar la existencia de niveles superiores de estrés en la adolescencia temprana y media, viéndose reducidos al final de la misma, cuestión que algunos autores atribuyen al desarrollo de estrategias de afrontamiento más eficaces (Williams y McGillicudy-De Lisi, 2000). Sin embargo, Byrne et al. (2007), en un estudio con adolescentes entre 13-18 años, constatan una relación significativa positiva modesta entre edad y la mayoría de dimensiones del estrés académico que evalúan. En consecuencia, de forma similar a lo destacado respecto a las diferencias en función del sexo de los adolescentes, estos trabajos también obtienen resultados divergentes entre contextos, dominios y situaciones específicas que evalúan (Seiffge-Krenke et al., 2009).

Jones and Hattie (1991), en un estudio con adolescentes escolarizados entre 13-18 años, constatan que la interacción sexo*edad es significativa en la dimensión relativa a miedo 
al fracaso, siendo las mujeres de mayor edad quienes muestran valores superiores frente a sus compañeros varones, mientras que los estudiantes con edades intermedias (nivel equivalente a $3-4^{\circ} \mathrm{ESO}$ ) presentan los niveles inferiores. En el polo opuesto, la dimensión presión familiar no evidencia relación significativa alguna con edad, sexo y grupo cultural. Por otro lado, también constatan la significatividad de las interacciones entre sexo y edad con el origen cultural de los estudiantes. Hankin et al.(2007), en un estudio longitudinal con adolescentes entre 13-16 años, señalan que las mujeres destacan mayor volumen de estresores que los varones en diversos contextos -especialmente en el interpersonal- pero no en el escolar, y que el estrés académico decrece a lo largo del curso en los niveles inferiores de edad $\left(1^{\circ}\right.$ y $2^{\circ}$ ESO) y se mantiene constante en los niveles superiores ( $\left.3^{\circ} \mathrm{ESO}\right)$. Espejo, Blanca, Fernández-Baena, y Trianes (2011), en un estudio en $1^{\circ}$ y $2^{\circ}$ de ESO, constatan la significatividad de la interacción sexo*edad, siendo las mujeres quienes muestran mayores manifestaciones emocionales, fisiológicas y conductuales del estrés, aunque exclusivamente en $2^{\circ}$ curso.

Kouzma y Kennedy (2004) enfatizan que el periodo de edad entre 17-18 años está poco representado en la investigación pese a las importantes decisiones que se adoptan en el mismo. En su estudio constatan que los niveles superiores de estrés se sitúan en el contexto académico -frente a familia, hogar y amigos-, siendo los principales estresores la obtención de buenos resultados, la realización de exámenes, la elección de estudios, el estudiar para los exámenes, así como las presiones por hacerlo bien. Desde esta perspectiva, los estudios centrados en la transición e incorporación a la universidad también constatan elevados niveles de estrés relacionados con las nuevas metodologías de enseñanza, aprendizaje y evaluación, con los cambios en los contextos y estilo de vida, así como con la inadecuada gestión del tiempo (García-Ros y Pérez-González, 2011).

Por último, destacar que numerosos estudios también se han centrado en analizar las variables que pueden moderar la relación entre el estrés académico y los efectos negativos que genera. Así, entre otros, su impacto está mediado por los mecanismos de afrontamiento de los estudiantes (Boekaerts, 2011; Häfner, Stock y Oberts, 2014), por su autoeficacia académica y expectativas de futuro (Suldo, Shaunessy y Hardesty, 2008), así como por su nivel de autodeterminación y numerosos factores del contexto educativo -p.ej., clima de aula(Raufelder, Kittler, Braun, Lätsch, Wilkinson y Hoferichter, 2014; Weinstein y Ryan, 2012).

De este modo, las discrepancias entre los resultados de las escasas investigaciones previas, junto a la ausencia de estudios que analicen en el contexto español el estrés académico a través de ESO en función del nivel educativo y sexo de los estudiantes, han promovido la realización de este trabajo, cuyos objetivos específicos son:

a) Identificar los principales estresores y niveles de estrés académico en ESO. La hipótesis de partida es que los niveles superiores de estrés se relacionarán con la sobrecarga de trabajo y con la realización de exámenes.

b) Analizar los efectos de nivel educativo y sexo sobre el estrés académico en esta etapa educativa. Se hipotetiza la existencia de efectos significativos de ambas variables, así como de la interacción entre las mismas. Así, aunque se prevé que las mujeres muestren 
mayores niveles de estrés y que se evidencie su reducción significativa a través de los cursos, los valores superiores se manifestarán en las mujeres en los cursos de incorporación (transición Educación Primaria-ESO) y finalización de etapa (transición ESOEnseñanza Secundaria Postobligatoria).

\section{Método}

\section{Participantes}

En el estudio participan 299 estudiantes de Enseñanza Secundaria Obligatoria en el curso académico 2013-2014. De forma más específica, 82 alumnos de $1^{\circ}$ curso (27.4\%), 94 alumnos de $2^{\circ}$ curso (31.4\%), 54 alumnos de $3^{\circ}$ curso (18.1\%) y 69 alumnos de $4^{\circ}$ curso (23.1\%), con una edad media de 13.49 años $(D T=1.24)$ y un rango de edad entre 12-18 años. 152 participantes eran mujeres $(50.8 \%)$ y 147 varones (49.2\%), con distribuciones similares por nivel educativo. Como método de obtención de la muestra se efectuó una selección al azar de 14 grupos aula de seis Institutos de Educación Secundaria de la provincia de Valencia, todos ellos de contextos socioeconómicos medio-bajos.

\section{Medidas}

Se aplicó el Cuestionario de Estrés Académico en Educación Secundaria-CEAS-, inicialmente elaborado para su evaluación en la transición a la universidad y adaptado a esta etapa educativa (García-Ros, Pérez-González, Pérez-Blasco, y Natividad, 2012). Se trata de un cuestionario tipo autoinforme que integra 19 ítems que describen situaciones académicas cotidianas potenciales generadoras de estrés. Los sujetos deben responder indicando el nivel de estrés generado por las mismas utilizando una escala de respuesta tipo Likert con valores de 1 a 5, en la que 1 significa "Nada de estrés" y 5 "Mucho estrés". El CEAS evalúa las cuatro dimensiones siguientes:

a) Realización de Tareas y Estudio -RTE-. Nivel de estrés que genera la realización de las tareas académicas y el estudio de las distintas materias. Integra seis items (p.ej., "elaborar trabajos que supongan buscar información y redactar"), con un promedio de 2.93 y una consistencia interna de.70 (en este trabajo de .72).

b) Sobrecarga Académica y Evaluaciones -SAE-. Nivel de estrés provocado por la percepción de falta de tiempo y sensación de estar desbordado por el volumen de trabajo a desarrollar, así como por la realización de exámenes. Integra 4 ítems (p. ej." "realización de exámenes"), con un promedio de 3.71 y una consistencia interna de .74 (en este estudio .73).

c) Futuro Académico -FA-. Estrés generado por la anticipación de situaciones y potenciales problemas académicos futuros, tales como la elección de itinerarios y optativas. Integra 4 items (e.g., "acabar la ESO en el plazo estipulado"), con un promedio de 3.30 y una consistencia interna de .77 (en este estudio. 71).

d) Relaciones Interpersonales -RI-. Nivel de estrés generado en las interacciones y conflictos interpersonales relacionados con el ámbito académico con profesores, compañeros o padres. Integra 5 items (e.g., "Conflictos familiares por los resultados académicos"), con un promedio de 2.81 y una consistencia interna de .66 (en este estudio .65). 
Adicionalmente, se aplicó un cuestionario dirigido a obtener la información sociodemográfica y académica de los estudiantes, considerando en este estudio el sexo y el nivel educativo.

\section{Procedimiento}

La aplicación de las pruebas se efectuó al inicio de la tercera evaluación del curso académico 2013-14 (principio del mes de abril), de forma colectiva en el aula y horario académico de los participantes. La participación fue voluntaria, solicitando previamente el consentimiento informado a las familias de los estudiantes para la participación en la investigación.

\section{Análisis}

Con el objetivo de determinar los posibles efectos del sexo y del nivel educativo sobre el estrés académico se efectuó un MANOVA factorial 2×2, en que las variables dependientes corresponden a las puntuaciones en las cuatro dimensiones del CEAS y las independientes al sexo y nivel académico de los estudiantes. A continuación, se efectuó un ANOVA para cada una de las dimensiones de estrés, aplicando posteriormente las pruebas post-hoc de Bonferroni. Todos los análisis fueron efectuados con el programa SPSS 19.0 para Windows.

\section{Resultados}

La tabla 1 presenta los descriptivos básicos de las puntuaciones en las distintas dimensiones de estrés académico en función del nivel educativo y sexo. Los valores superiores se evidencian en $S A E$ seguido por $F A$ en todos los niveles y en ambos sexos, presentado puntuaciones promedio superiores a la media teórica de la escala de respuesta. En el lado opuesto, las puntuaciones inferiores se obtienen en RTE y RI. Las situaciones que generan mayor estrés en todos los cursos y en ambos sexos son "la sobrecarga académica -tener muchos exámenes y trabajos obligatorios-" $(M=4.12, D T=1.11)$, "la falta de tiempo para cumplir con las actividades" $(M=3.76, D T=1.20)$ y "la realización de exámenes" $(M=3.60, D T$ $=1.19$ ), todas ellas integradas en $S A E$. Por otro lado, las situaciones en que un mayor por-

Tabla 1. Medias y desviaciones típicas (entre paréntesis) de las dimensiones de estrés académico en función de sexo y nivel educativo

\begin{tabular}{|c|c|c|c|c|c|c|c|c|c|c|c|c|c|c|c|}
\hline & \multicolumn{3}{|c|}{ 1er curso } & \multicolumn{3}{|c|}{$2^{\circ}$ curso } & \multicolumn{3}{|c|}{ 3er curso } & \multicolumn{3}{|c|}{$4^{0}$ curso } & \multicolumn{3}{|c|}{ Total } \\
\hline & Hombres & Mujeres & Total & Hombres & Mujeres & Total & Hombres & Mujeres & Total & Hombres & Mujeres & Total & Hombres & Mujeres & Total \\
\hline RTE & $\begin{array}{c}2.98 \\
(1.01)\end{array}$ & $\begin{array}{c}3.11 \\
(0.81)\end{array}$ & $\begin{array}{c}3.05 \\
(0.91)\end{array}$ & $\begin{array}{c}2.57 \\
(0.80)\end{array}$ & $\begin{array}{c}2.85 \\
(0.83)\end{array}$ & $\begin{array}{c}2.72 \\
(0.83)\end{array}$ & $\begin{array}{c}2.80 \\
(0.76)\end{array}$ & $\begin{array}{c}2.71 \\
(0.79)\end{array}$ & $\begin{array}{c}2.75 \\
(0.77)\end{array}$ & $\begin{array}{c}2.15 \\
(0.80)\end{array}$ & $\begin{array}{c}2.82 \\
(0.86)\end{array}$ & $\begin{array}{c}2.46 \\
(0.89)\end{array}$ & $\begin{array}{c}2.62 \\
(0.90)\end{array}$ & $\begin{array}{c}2.89 \\
(0.83)\end{array}$ & $\begin{array}{c}2.76 \\
(0.88)\end{array}$ \\
\hline SAE & $\begin{array}{c}3.76 \\
(1.03)\end{array}$ & $\begin{array}{c}3.63 \\
(0.77)\end{array}$ & $\begin{array}{c}3.69 \\
(0.90)\end{array}$ & $\begin{array}{c}3.58 \\
(0.83)\end{array}$ & $\begin{array}{c}3.87 \\
(0.92)\end{array}$ & $\begin{array}{c}3.73 \\
(0.89)\end{array}$ & $\begin{array}{c}3.67 \\
(0.87)\end{array}$ & $\begin{array}{r}3.69 \\
(0.67)\end{array}$ & $\begin{array}{c}3.68 \\
(0.91)\end{array}$ & $\begin{array}{c}3.04 \\
(0.93)\end{array}$ & $\begin{array}{c}3.88 \\
(0.78)\end{array}$ & $\begin{array}{c}3.42 \\
(0.96)\end{array}$ & $\begin{array}{c}3.51 \\
(0.96)\end{array}$ & $\begin{array}{c}3.77 \\
(0.86)\end{array}$ & $\begin{array}{c}3.64 \\
(0.92)\end{array}$ \\
\hline FA & $\begin{array}{c}3.46 \\
(1.09)\end{array}$ & $\begin{array}{c}3.32 \\
(1.06)\end{array}$ & $\begin{array}{r}3.39 \\
(1.07)\end{array}$ & $\begin{array}{c}2.92 \\
(1.02)\end{array}$ & $\begin{array}{c}3.28 \\
(0.94)\end{array}$ & $\begin{array}{c}3.12 \\
(0.99)\end{array}$ & $\begin{array}{c}3.12 \\
(0.83)\end{array}$ & $\begin{array}{r}3.46 \\
(1.15)\end{array}$ & $\begin{array}{c}3.29 \\
(1.00)\end{array}$ & $\begin{array}{c}2.66 \\
(0.92)\end{array}$ & $\begin{array}{c}3.65 \\
(0.98)\end{array}$ & $\begin{array}{c}3.12 \\
(1.06)\end{array}$ & $\begin{array}{c}3.04 \\
(1.01)\end{array}$ & $\begin{array}{c}3.40 \\
(1.02)\end{array}$ & $\begin{array}{c}3.22 \\
(1.03)\end{array}$ \\
\hline RI & $\begin{array}{c}2.96 \\
(0.96)\end{array}$ & $\begin{array}{c}2.98 \\
(0.88)\end{array}$ & $\begin{array}{c}2.97 \\
(0.91)\end{array}$ & $\begin{array}{c}2.72 \\
(0.84)\end{array}$ & $\begin{array}{c}2.89 \\
(0.87)\end{array}$ & $\begin{array}{c}2.81 \\
(0.86)\end{array}$ & $\begin{array}{c}2.81 \\
(0.80)\end{array}$ & $\begin{array}{c}2.39 \\
(0.87)\end{array}$ & $\begin{array}{c}2.60 \\
(0.86)\end{array}$ & $\begin{array}{c}2.02 \\
(0.73)\end{array}$ & $\begin{array}{c}2.56 \\
(0.74)\end{array}$ & $\begin{array}{c}2.27 \\
(0.78)\end{array}$ & $\begin{array}{c}2.62 \\
(0.91)\end{array}$ & $\begin{array}{c}2.76 \\
(0.87)\end{array}$ & $\begin{array}{c}2.69 \\
(0.89)\end{array}$ \\
\hline
\end{tabular}

Nota . RTE = Realización de Tareas y Estudio; SAE = Sobrecarga Académica y Evaluaciones; FA = Futuro Académico; RI = Relaciones interpersonales 
centaje de estudiantes manifiestan haber experimentado niveles elevados de estrés corresponden a sobrecarga académica (47.6\% de los estudiantes), falta de tiempo para cumplir las actividades (35.6\%), acabar la ESO en los plazos estipulados (34.1\%), presión familiar por obtener buenos resultados (30\%) y realización de exámenes (30\%).

Los resultados del MANOVA muestran la existencia de efectos significativos -aunque con un tamaño del efecto reducido- sobre el estrés académico de las variables sexo, $\Lambda=.96, F$ $(4,288.000)=3.36, p<.01, \eta^{2}=.05, \mathrm{y}$ nivel educativo, $\Lambda=.86, F(12,762.268)=3.67, p$ $<.001, \eta^{2}=.05$, así como de la interacción sexo*nivel educativo, $\Lambda=.92, F(12,762.268)$ $=2.06, p<.02, \eta^{2}=.03$.

Con el objetivo de clarificar los resultados del MANOVA se efectuaron los correspondientes ANOVAs para cada dimensión de estrés, cuyos resultados se presentan en la tabla 2. Se constata la significatividad de los efectos principales de la variable sexo en tres de las dimensiones evaluadas $-R T E$, $S A E$ y $F A$-y del nivel educativo en dos de ellas $-R T E$ y $R I$-, resultando también significativa la interacción sexo*nivelen tres dimensiones $-S A E, F(3$, $291)=4.15, p<.01, \eta^{2}=.04 ; F A, F(3,291)=3.99, p<.01, \eta^{2}=.04 ; R I, F(3,291)=3.43$, $p<.02, \eta^{2}=.04-$, y marginalmente significativa para $R T E, F(3,291)=2.35, p<.07, \eta^{2}=$ .02. En este último caso, son significativos los efectos principales tanto de sexo, $F(1,291)=$ $6.31, p<.01, \eta^{2}=.02$, como de nivel educativo $F(3,291)=5.70, p<.001, \eta^{2}=.06$.

Tabla 2. ANOVAS sobre las dimensiones de estrés académico: Efectos principales e interacción entre sexo y nivel educativo

\begin{tabular}{ccccccccccccc}
\hline & \multicolumn{4}{c}{ Sexo } & \multicolumn{4}{c}{ Nivel Educativo } & \multicolumn{3}{c}{ Sexo x Nivel educativo } \\
\cline { 2 - 13 } & $F$ & $\mathrm{gl}$ & $p$ & $\eta^{2}$ & $F$ & $\mathrm{gl}$ & $p$ & $\eta^{2}$ & $F$ & $\mathrm{gl}$ & $p$ & $\eta^{2}$ \\
\cline { 2 - 13 } RTE & 6.31 & 1 & .02 & .02 & 5.70 & 3 & .001 & .06 & 2.34 & 3 & .07 & .02 \\
SAE & 5.91 & 1 & .02 & .02 & 1.38 & 3 & .25 & .01 & 4.15 & 3 & .01 & .04 \\
FA & 10.57 & 1 & .001 & .03 & 1.39 & 3 & .25 & .02 & 3.99 & 3 & .01 & .04 \\
RI & 0.62 & 1 & .43 & .01 & 8.97 & 3 & .001 & .07 & 3.43 & 3 & .02 & .04 \\
\hline
\end{tabular}

Nota . RTE = Realización de Tareas y Estudio; SAE = Sobrecarga Académica y Evaluaciones; FA = Futuro Académico; RI = Relaciones interpersonales

De forma más específica, los análisis a posteriori indicaron que en $S A E$ las mujeres presentan mayores niveles de estrés que los varones en $4^{\circ}$ curso $(p<.001)$ y que los varones muestran niveles significativamente superiores en $1^{\circ}$ respecto a $4^{\circ}$ curso $(p<.01)$, así como entre $2^{\circ}$ y $4^{\circ}(p<.03)$, mientras que las mujeres no evidencian diferencia significativa alguna a través de toda la etapa. Resultados similares se obtienen en $F A$, dado que las mujeres presentan valores superiores a los varones en $4^{\circ}$ curso $(p<.01)$ y niveles homogéneos a través de la etapa, mientras que los varones muestran niveles superiores en $1^{\circ}$ respecto a $4^{\circ}(p<.001)$.

En $R I$ las mujeres también presentan niveles significativamente superiores a los varones en $4^{\circ}$ curso $(p<.01)$, los varones muestran puntuaciones inferiores en $4^{\circ}$ frente al resto de niveles previos(respecto a $2^{\circ}$ y 3 er curso, $p<.001$; respecto a primer curso, $p<.01$ ) y las 
mujeres sólo muestran diferencias significativas entre $1^{\circ}-3^{\circ}$ curso $(p<.05)$.Por último, en RTE las mujeres manifiestan mayores niveles de estrés que los varones $(p<.05)$ y se observan diferencias significativas entre $1^{\circ}-4^{\circ}$ curso $(p<.05)$ en el sentido de reducción del estrés académico en esta dimensión.

\section{Conclusiones}

Coincidiendo con la primera hipótesis de trabajo, los resultados muestran que las principales fuentes de estrés académico en esta etapa se relacionan con la sobrecarga académica y la realización de exámenes, así como con las preocupaciones por el futuro académico. Las dimensiones relativas a realización de tareas y estudio, así como con las relaciones interpersonales -compañeros, profesores y padres- presentan niveles promedio menores, incluso ligeramente inferiores a la media teórica de la escala de respuesta.

De hecho, las situaciones que generan mayor estrés en todos los niveles y en ambos sexos son "la sobrecarga académica (tener muchos exámenes y trabajos obligatorios)", "la falta de tiempo para cumplir con las actividades" y "la realización de exámenes", todas ellas integradas en $S A E$. Por otro lado, en todas las situaciones académicas planteadas se evidencia un porcentaje significativo de sujetos (rango 6.3 - 47.6\%) que manifiesta haber experimentado mucho estrés, constatándose los porcentajes superiores en sentirse desbordado por la sobrecarga de trabajo, la falta de tiempo para realizar las actividades, el concluir la ESO en los plazos estipulados, la presión familiar por obtener buenos resultados y la realización de exámenes. De forma relevante para la prevención e intervención educativa en este ámbito, también se constata que existe un volumen importante de sujetos que experimentan niveles elevados de estrés incluso en las situaciones con valores promedio inferiores $(6.3 \%$ de sujetos en "realización de trabajos que suponen búsqueda de información e integración de la misma"; 7.6\% en "trabajar con compañeros en la realización de tareas"; $8.2 \%$ en "competitividad con los compañeros").

Los resultados también evidencian la relevancia del nivel educativo y del sexo en las dimensiones de estrés académico consideradas. De forma más específica, se observan patrones idénticos en $S A E$ y $F A$, constatando la significatividad de la interacción sexo*nivel educativo, siendo las mujeres quienes presentan mayor estrés en $4^{\circ}$ curso-previo a la incorporación a la enseñanza postobligatoria- respecto a los varones, pero no en los precedentes. Adicionalmente, los varones evidencian un descenso del estrés con el tiempo -alcanzando la significatividad estadística entre $1^{\circ}-4^{\circ}$ curso-, mientras que las mujeres muestran niveles homogéneos a través de toda la ESO. Los resultados también son similares en RI, obteniendo diferencias entre sexos solo en $4^{\circ}$ curso, constatando la homogeneidad entre niveles educativos en las mujeres y observando un descenso significativo entre los varones en el último curso respecto a los precedentes. En lo que respecta a $R T E$, las mujeres presentan niveles superiores de estrés respecto a los varones -especialmente en $2^{\circ}$ y $4^{\circ}$ curso-, evidenciándose su reducción significativa en ambos sexos en la comparación $1^{\circ}-4^{\circ}$ curso.

En consecuencia, los resultados apoyan parcialmente la segunda hipótesis de trabajo, que preveía la existencia efectos principales significativos del sexo y del nivel educativo sobre el estrés académico, así como la significatividad de la interacción sexo*nivel educativo. De

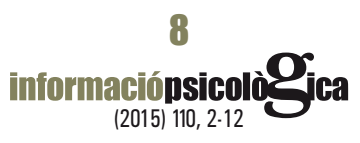


forma más específica, las mujeres presentan mayores niveles de estrés que los varones en la dimensión de realización de tareas y estudio - $R T E$-, cuestión que destaca su mayor preocupación por desarrollar las actividades de aprendizaje y estudio de forma satisfactoria (Murberg y Bru, 2004), y observándose también una reducción significativa entre el curso inicial y final de la etapa congruente con el desarrollo de repertorios de estrategias de aprendizaje más amplios y efectivos (Wigfield, Byrnes y Eccles, 2006).

Sin embargo, pese a que también se observan efectos principales significativos de sexo en las dimensiones de sobrecarga académica y realización de exámenes $-S A E$ - y de preocupaciones por el futuro $-F A-$, así como del nivel educativo en la dimensión relativa a relaciones interpersonales $-R I$-, en los tres casos es también significativa la interacción sexo*nivel educativo, de modo que la interpretación de resultados se supedita a la misma. De este modo, aunque las mujeres presentan mayor estrés que los varones en las tres dimensiones, sólo resulta significativamente superior en el último curso de la etapa, previo a la incorporación a la enseñanza postobligatoria. Adicionalmente, se observa una reducción significativa del estrés entre el curso inicial y final de etapa exclusivamente entre los varones, mientras que las mujeres presentan valores homogéneos a través de los cursos. Estos resultados coincidirían con los obtenidos en trabajos previos que analizan los efectos del sexo sobre el estrés académico en la transición entre etapas educativas, ya sea entre enseñanza obligatoria y postobligatoria (Jones y Hattie, 1991) o en el acceso a la universidad (p.ej., Kouzma y Kennedy, 2004). Sin embargo, mención especial merece el que no se hayan observado diferencias en función del sexo en el año de incorporación a la ESO, pese a los notables cambios a los que se enfrentan los estudiantes (Rice et al., 2011). Esta cuestión deberá ser analizada con mayor profundidad en estudios posteriores, ya que podría ser interpretada en términos de que varones y mujeres pueden presentar niveles similares de estrés al incorporarse a la enseñanza secundaria, de los efectos de las estrategias y programas que desarrollan los centros con el objetivo de facilitar la transición entre Enseñanza Primaria y Secundaria (p.ej., utilizar como criterio de agrupamiento el centro de primaria de procedencia o reducir el número de profesores por grupo trabajando por ámbitos), o por el momento específico en que se evalúa el estrés académico (p.ej., en las fases iniciales del curso o con el mismo ya muy avanzado -tal como se ha efectuado en este trabajo- en que los estudiantes ya están familiarizados con los cambios respecto a Enseñanza Primaria).

En cualquier caso, en la dimensión del estrés académico relacionada con la realización de actividades y con el estudio, los resultados apoyan la mayor vulnerabilidad de las mujeres adolescentes frente a los varones, así como la presencia de mayores niveles de estrés en el curso de incorporación a la etapa frente a cursos más avanzados, cuestiones a las que deberán prestar especial atención tanto los orientadores como el profesorado de las distintas materias. Y, por otro lado, en lo que respecta al estrés relacionado con la sobrecarga de tareas y realización de exámenes, con la anticipación de problemas académicos futuros y con las relaciones interpersonales, las mujeres también presentan una mayor vulnerabilidad al estrés en el último curso de la etapa, mientras que sólo los varones evidencian una reducción significativa de sus niveles de estrés a través de los cursos, cuestiones también a considerar 
en la planificación de programas de intervención tanto a nivel general como individual en esta etapa educativa.

Centrándonos en las repercusiones de este trabajo, los resultados destacan que la intervención en gestión del estrés parece resultar útil para el conjunto de estudiantes y en todos los niveles de la Educación Secundaria Obligatoria. Adicionalmente, que resulta especialmente relevante centrar los esfuerzos de la intervención en el desarrollo de las habilidades de gestión del tiempo (sobrecarga de trabajo), en el afrontamiento de las situaciones de evaluación (realización de exámenes), así como en los procesos de toma de decisiones y en el establecimiento de metas y objetivos académicos realistas (futuro académico). Cuestión que no supone minusvalorar la importancia de otras áreas de intervención, dado el volumen significativo de sujetos que experimentan niveles elevados de estrés en las mismas, tales como el afrontamiento y resolución de problemas en las relaciones con el profesorado y con los compañeros o la presión familiar por la obtención de buenos resultados. Así, dado que los problemas y conflictos en las relaciones interpersonales constituyen un claro factor de riesgo para el deterioro del rendimiento y del funcionamiento académico (Hoferichter, Raufelder y Eid, 2014; Liu y Lu, 2011b), este área de intervención también deberá ocupar un lugar preeminente, aunque presumiblemente considerando modalidades de intervención más individualizadas o centradas en el profesorado y en la gestión del aula (Natvig, Albrektsen, Anderssen, y Qvarnstrøm, 1999). En esta misma línea, destacar la importancia de distintos factores organizativos que pueden resultar especialmente relevantes para reducir los niveles de estrés que experimentan los estudiantes, tales como la coordinación del profesorado en la asignación de tareas y en la planificación del calendario de exámenes, el diseño de entornos de aprendizaje y la utilización de metodologías de enseñanza y evaluación centradas en el estudiante, o la mejora de los sistemas de apoyo ante las dificultades que puedan surgir en la realización de las tareas y en el estudio de las materias.

La determinación de las principales fuentes y situaciones académicas que generan mayores niveles de estrés en esta etapa educativa, junto a la constatación de los efectos significativos del nivel educativo y sexo sobre las dimensiones analizadas, no impiden destacar las limitaciones de este trabajo que deberán ser consideradas en futuras investigaciones. Así, su carácter transversal no permite analizar posibles patrones de desarrollo a lo largo de la etapa y en las distintas dimensiones del estrés consideradas, cuestión que destaca la necesidad de primar los estudios de carácter longitudinal sobre esta temática. Por otro lado, también resultaría de interés considerar posibles variables mediadoras que puedan modular los efectos del estrés y permitan ajustar con mayor precisión la intervención a las características y necesidades de los estudiantes. Para finalizar, considerar un rango más amplio de edad permitiría incorporar en la investigación distintos itinerarios y niveles de la enseñanza postobligatoria que, junto a la evaluación del estrés en distintos momentos a lo largo del curso académico, supondrían un conocimiento más profundo y de mayor utilidad para la intervención en este ámbito. 


\section{Referencias}

Antonson, C., Thorsén, F., Sundquist, K., y Sundquist, J. (2014). Stress-Related Symptoms in Swedish Adolescents: A Study in Two Upper Secondary Schools. Journal of Educational and Developmental Psychology, 4, 47-64. http://dx.doi.org/10.5539/jedp.v4n2p65

Boekaerts, M. (2011). Emotions, emotion regulation, and self regulation of learning. In B.J. Zimmerman y D.H. Schunk (Eds.): Handbook of self-regulation of learning and performance (pp.408-425). New York: Routledge.

Byrne, D. G., Davenport, S. C., y Mazanov, J. (2007). Profiles of adolescent stress: The development of the adolescent stress questionnaire (ASQ). Journal of adolescence, 30, 393-416. http://dx.doi.org/10.1016/j.adolescence.2006.04.004

De Anda, D., Baroni, S., Boskin, L., Buchwald, L., Morgan, J., Ow, J., ...y Weiss, R. (2000). Stress, stressors and coping among high school students. Children and youth services review, 22, 441-463. http://dx.doi. org/10.1016/S0190-7409(00)00096-7

Espejo, M. E., Blanca, M. J., Fernández-Baena, F. J., y Torres, M. V. T. (2011). Adaptación española de la escala de manifestaciones de estrés del Student Stress Inventory (SSI-SM). Psicothema, 23, 475-485.

García-Ros, R., y Pérez-González, F. (2011). Validez predictiva e incremental de las habilidades de autorregulación sobre el éxito académico en la universidad. Revista de Psicodidáctica, 16, 231-250.

García-Ros, R., Pérez-González, F., Pérez-Blasco, J., y Natividad, L. A. (2012). Evaluación del estrés académico en estudiantes de nueva incorporación a la universidad [Academic stress in First-YearCollegestudents]. Revista latinoamericana de psicología, 44, 143-154.

Grant, K. E., Compas, B. E., Thurm, A. E., McMahon, S. D., Gipson, P.Y., Campbell, A. J., ...y Westerholm, R. I. (2006). Stressors and child and adolescent psychopathology: Evidence of moderating and mediating effects. Clinical psychology review, 26, 257-283. http://dx.doi.org/10.1016/j.cpr.2005.06.011

Häfner, A., Stock, A., y Oberst, V. (2014). Decreasing students' stress through time management training: an intervention study. European Journal of Psychology of Education, 30, 81-94. http://dx.doi.org/10.1007/ $\underline{\mathrm{s} 10212-014-0229-2}$

Hankin, B. L., Mermelstein, R., y Roesch, L. (2007). Sex differences in adolescent depression: Stress exposure and reactivity models. Child development,78, 279-295. http://dx.doi.org/10.1111/j.1467-8624.2007.00997.x

Hoferichter, F., Raufelder, D., y Eid, M. (2014). The mediating role of socio-motivational relationships of perceived stress, neuroticism, and test anxiety among adolescent students. Psychology in the Schools, 51, 736752. http://dx.doi.org/10.1002/pits.21778

Jones, R. W., y Hattie, J. A. (1991, April). Academic Stress amongst Adolescents: An Examination by Ethnicity, Grade, and Sex.Paper presented at the Annual Conference of the New England Educational Research Organization, Portsmout, $\mathrm{NH}$.

Kaplan, D. S., Liu, R. X., y Kaplan, H. B. (2005). School related stress in early adolescence and academic performance three years later: the conditional influence of self expectations. Social Psychology of Education, 8, 3-17. http://dx.doi.org/10.1007/s11218-004-3129-5

Kouzma, N. M., y Kennedy, G.A. (2004). Self-reported sources of stress in senior high-school students. Psychological reports, 94 (1), 314-316. http://dx.doi.org/10.2466/pr0.94.1.314-316

Liu, Y., y Lu, Z. (2011a). The Chinese high school student's stress in the school and academic achievement. Educational Psychology, 31, 27-35. http://dx.doi.org/10.1080/01443410.2010.513959

Liu, Y., y Lu, Z. (2011b). Longitudinal analysis of Chinese high school student's stress in school and academic achievement. Educational Psychology, 31, 723-729. http://dx.doi.org/10.1080/01443410.2011.600245

Murberg, T. A., y Bru, E. (2004). School-related stress and psychosomatic symptoms among Norwegian adolescents. School psychology international, 25, 317-332. http://dx.doi.org/10.1177/0143034304046904 
Natvig, G. K., Albrektsen, G., Anderssen, N., y Qvarnstrøm, U. (1999). School-related Stress and Psychosomatic Symptoms Among School Adolescents. Journal of school health, 69, 362-368. http://dx.doi. org/10.1111/j.1746-1561.1999.tb06430.x

Raufelder, D., Kittler, F., Braun, S. R., Lätsch, A., Wilkinson, R. P., y Hoferichter, F. (2014). The interplay of perceived stress, self-determination and school engagement in adolescence. School Psychology International, 35, 405-420. http://dx.doi.org/10.1177/0143034313498953

Rayle, A. D., y Chung, K. Y. (2007). Revisiting first-year college students' mattering: Social support, academic stress, and the mattering experience. Journal of College Student Retention: Research, Theory and Practice, 9, 21-37. http://dx.doi.org/10.2190/X126-5606-4G36-8132

Rice, F., Frederickson, N., y Seymour, J. (2011). Assessing pupil concerns about transition to secondary school. British Journal of Educational Psychology, 81, 244-263. http://dx.doi.org/10.1348/000709910X519333

Seiffge-Krenke, I., Aunola, K., y Nurmi, J. E. (2009). Changes in stress perception and coping during adolescence: The role of situational and personal factors. Child development, 80, 259-279. http://dx.doi.org/10.1111/ j.1467-8624.2008.01258.x

Suldo, S. M., Shaunessy, E., y Hardesty, R. (2008). Relationships among stress, coping, and mental health in highachieving high school students. Psychology in the Schools, 45, 273-290. http://dx.doi.org/10.1002/ pits.20300

Weinstein, N., y Ryan, R. M. (2011). A self-determination theory approach to understanding stress incursion and responses. Stress and Health, 27,4-17. http://dx.doi.org/10.1002/smi.1368

Wigfield, A., Byrnes, J. P., y Eccles, J. S. (2006). Development during early and middle adolescence. In P. A. Alexander y P. H. Winne (Eds.), Handbook of educational psychology (pp. 87-113). London: LEA.

Williams, K., y McGillicuddy-De Lisi, A. (2000). Coping strategies in adolescents. Journal of Applied Developmental Psychology, 20, 537-549. http://dx.doi.org/10.1016/S0193-3973(99)0002 